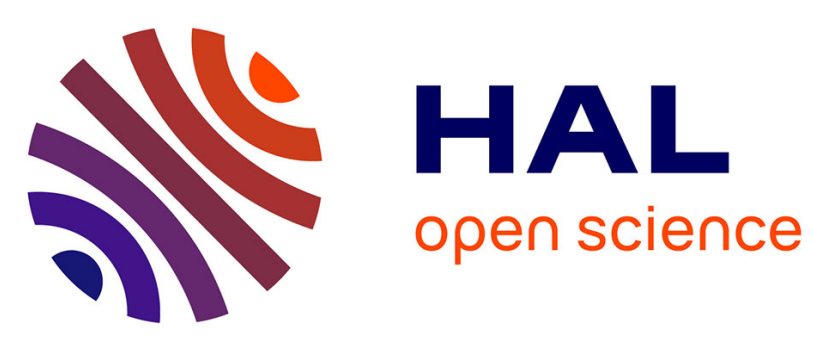

\title{
Serial and Parallel Active Decoupling Characterization Using RF MEMS Switches for Receiver Endoluminal Coils at $1.5 \mathrm{~T}$
}

Hamza Raki, Kevin Tse Ve Koon, Isabelle Saniour, Henri Souchay, Simon A Lambert, Fraser Robb, Olivier Beuf

\section{To cite this version:}

Hamza Raki, Kevin Tse Ve Koon, Isabelle Saniour, Henri Souchay, Simon A Lambert, et al.. Serial and Parallel Active Decoupling Characterization Using RF MEMS Switches for Receiver Endoluminal Coils at 1.5 T. IEEE Sensors Journal, 2020, 20 (18), pp.10511-10520. 10.1109/JSEN.2020.2995055 . hal-02978611

\section{HAL Id: hal-02978611 \\ https://hal.science/hal-02978611}

Submitted on 24 Nov 2020

HAL is a multi-disciplinary open access archive for the deposit and dissemination of scientific research documents, whether they are published or not. The documents may come from teaching and research institutions in France or abroad, or from public or private research centers.
L'archive ouverte pluridisciplinaire HAL, est destinée au dépôt et à la diffusion de documents scientifiques de niveau recherche, publiés ou non, émanant des établissements d'enseignement et de recherche français ou étrangers, des laboratoires publics ou privés. 


\title{
Serial and Parallel Active Decoupling Characterization Using RF MEMS Switches for Receiver Endoluminal Coils at 1.5T
}

\author{
Hamza Raki, Kevin Tse Ve Koon, Isabelle Saniour, Henri Souchay, Simon A. Lambert, \\ Fraser Robb and Olivier Beuf
}

\begin{abstract}
MEMS (Micro Electro Mechanical System) switches were assessed and compared to PIN diode in fulfilling the task of active decoupling of Receiver Endoluminal Coils (RECs). Three prototype RECs with the PIN diode in parallel ( $P$ PIN), MEMS in parallel ( $p M E M S$ ) and MEMS in series (sMEMS) with the REC loop were built. Quality factors (Q-values), decoupling efficiency and switching delays were characterized on bench and Signal-to-Noise Ratios (SNRs) established on images at 1.5 T. Q-values were equal to $62.5,41.2$ and 65.1 for pPIN, sMEMS and pMEMS, respectively. In the decoupled state, reflection coefficients $S_{11}$ and $S_{21}$ at
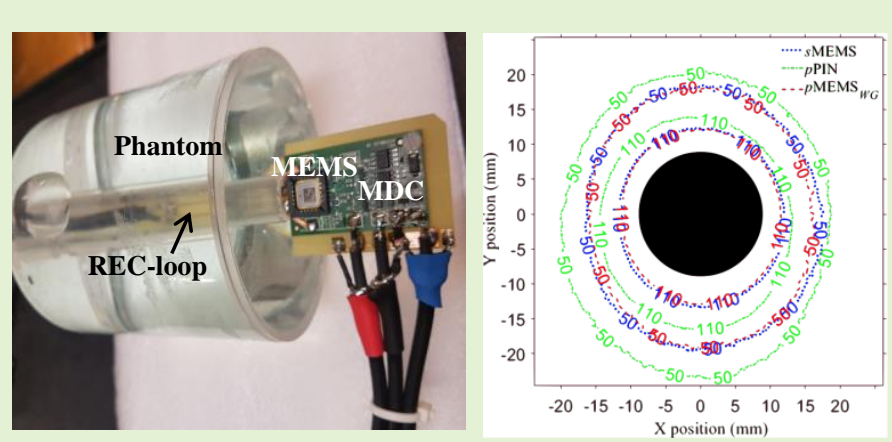
resonance frequency both indicated proper decoupling. Switching delays were less than $0.7 \mu s$ and $10 \mu s$ for $p P I N$ and MEMS RECs, respectively. Decoupling/coupling delays of MEMS remained compatible with most Magnetic Resonance (MR) clinical applications. For all prototypes, MR images displayed no signal saturation and similar elliptical image sensitivity patterns. No artifacts due to active decoupling failure were observed. Mean SNR values obtained with pMEMS REC were higher than those obtained with sMEMS REC but lower than with $p$ PIN REC because of the use of additional instrumentation to render the scanner compatible with the MEMS utilization. MEMS in parallel are an interesting alternative to PIN diode for decoupling and could lead to better SNR with a compatible MR system (dedicated control signal). The MEMS in series can be used for both decoupling and reconfiguration of the REC loop geometry for colon wall examination.
\end{abstract}

Index Terms- Active decoupling, endoluminal coils, MEMS, MR switch.

\section{INTRODUCTION}

$\mathrm{M}$ agnetic Resonance Imaging (MRI) is a common modality used for medical diagnosis of abdominal and intestinal diseases. In the case of colorectal cancer, which is one of the most frequent and fatal cancers worldwide [1]-[5], colonoscopy investigation remains the gold standard. One intrinsic limitation of this optical imaging technique is the low

Manuscript submitted September 20, 2019, resubmitted January 28, 2020 and revised March 23, 2020. Authors would like to thank GE Healthcare, Inc. and ANRT for funding this project. This work was conducted in the scope of the LABEX PRIMES of University of Lyon (ANR-11-LABX-0063), within the program "Investissements d'Avenir" (ANR-11-IDEX-0007) operated by the French National Research Agency (ANR).

Hamza Raki, Kevin Tse Ve Koon, Isabelle Saniour, Simon A Lambert and Olivier Beuf are with the Univ Lyon, INSA-Lyon, Université Claude Bernard Lyon 1, UJM-Saint Etienne, CNRS, Inserm, CREATIS UMR 5220, U1206, F-69621, Villeurbanne, France (e-mail: hamza.raki@creatis.insa-lyon.fr; Kevin.Tsevekoon@creatis.insalyon.fr; isabellesaniour@live.com; Simon.Lambert@creatis.insa-Iyon.fr; Olivier.Beuf@creatis.insa-lyon.fr).

Henri Souchay and Hamza Raki (e-mail: Henri.SOUCHAY@med.ge.com; hamza.raki@gmail.com) are with the GE Healthcare, Buc 78530 France.

Fraser Robb is with the GE Healthcare, Ohio 44202 USA (e-mail: fraser.robb@ge.com). penetration depth, which can result in tumor growing within the bowel wall being detected only at a late stage. This limitation could be overcome by a high spatial resolution (inplane pixel size $<100 \mu \mathrm{m}$ and $2-\mathrm{mm}$ slice thickness) MRI examination.

MR arrays of external receiver radiofrequency (RF) coils are commonly used for abdominal imaging with improved Signal-to-Noise Ratio (SNR) owing to the combination of small surface coils that provide higher sensitivity [6]-[8]. Unfortunately, the gain in SNR is not sufficient to depict the thin bowel and colon wall in deep regions located relatively far from the body surface, thus making analysis of the colon wall and subsequent colorectal cancer staging difficult.

For this purpose, early works [9]-[12] have demonstrated the feasibility and value of Receiver Endoluminal Coils (RECs) close to the region of interest (ROI). A simple REC design consists of a very small surface coil with an adapted size and geometry so as to fit the lumen being investigated. In the case of colon examinations, the favored geometry is a rectangular loop with a width somewhat smaller than the diameter of the colon. In this configuration, the REC displays a cylindrical sensitivity pattern (with respect to its long axis) 
with a very high SNR close to the loop, enabling a decreased voxel size. The sensitivity decreases rapidly with the distance to the long axis [13] which is, however, sufficient to enable high-resolution imaging of the first centimeters of the colon wall and thus visualization of its different layers. RECs have been used in vivo on rabbit [14] and mouse [15], [16] colon walls. The REC is used in combination with the whole-body RF transmitter coil in order to provide a well-transmitted RF pulse uniformity over the imaging volume [17]. Both coils must hence be decoupled during the transmission phase in order to avoid any mutual induction and the subsequent nonuniform $\mathrm{B}_{1}$ magnetic field in the vicinity of the receiver loop coil. Therefore, a decoupling circuit is mandatory to avoid uncontrolled and spatially dependent image contrast. Another negative consequence is the local increase in the Specific Absorption Rate (SAR) that could lead to patient safety problems [18].

To this end, several electronic switching components enable this active decoupling. PIN diodes are the most popular components used as RF switches in the majority of clinical systems. The main advantages are their compatibility with a magnetic environment (non-magnetic packaging), the small size (a few millimeters) and a switching delay below $1 \mu \mathrm{s}$.

Other components such as MR-compatible memory resistive elements (Memristors) have been used [19]. The principle resides in applying different DC control voltages to switch between two resistive states during transmit and receive modes. The particularity of this technology is that the Memristor, unlike the PIN diode, can memorize and retain the resistive state information after the control signal is removed. It is particularly relevant in multinuclear MR coils. Other methods based on optical components have been proposed in the literature mainly founded on photoelectronic devices such as the photoresistor [20], photodiode [21], [22], combined photodiodes and a PIN diode [23], PhotoMOS [24], or MOSFET with optically isolated [25] devices. The optical decoupling solutions have the advantage of using nongalvanic transmission means thereby avoiding induced current in the shield and increasing patient safety [26]. Finally, low noise preamplifiers have been used for the decoupling between loops of the coil in the case of multiple-element array coils [27]-[29].

A few years ago, Micro ElectroMechanical System (MEMS) switches were introduced by GE Healthcare, Inc. [30] to act as MR-compatible switches (Fig. 1-a and b). As can be seen in Figure 1 (c and d), the MEMS as a switch has two working states: open and closed. The open state electrically corresponds to a small capacitance $(1.5-2 \mathrm{pF})$. The closed state is electrically equivalent to a very small parasitic resistance $(0.5-1 \Omega)$. Owing to their high electrical isolation (approximately $10 \mathrm{M} \Omega$ ) and their MR compatibility, MEMS ("MEMS" will be used to denote "MEMS switch" hereafter) were successfully used as switches. MEMSs were used to open or close portions of metallic conductors for rapid reconfiguration of external two-channel array coil geometry as was done for spine and torso MRI [31]. MEMSs have also been used as an alternative solution to the traditional passive decoupling strategy in the case of double-tuned RF coil designs used, for example, for sodium imaging. A proton coil is still required for co-registration purposes and the decoupling of the coils is performed with MEMSs by shifting the resonance frequencies. The MEMS-based solution demonstrated comparable results to the PIN diode and better performance than the trap circuit [32]. In the same context, a study based on four-element fixed phased transmit-receive coils used MEMS to switch between $1 \mathrm{H}(64 \mathrm{MHz})$ and $19 \mathrm{~F}$ $(60 \mathrm{MHz})$ resonance frequencies for lung imaging [33]. In a design of integrated parallel reception, excitation and shimming coil arrays, MEMSs were used to reduce the cost and complexity of the design by employing a single DC supply with an adaptive distribution of the DC current (instead of using "N" DC supplies for "N" shim loops) and thus achieving high RF shimming performances (high localized $\mathrm{B}_{0}$ homogeneity) with no SNR loss [34]. Finally, MEMSs were also used for both the control of an array of primary coils and the automatization of the impedance matching network of an MRI-compatible wireless power transfer system used on a wearable wireless receive coil array [35].

Endoluminal MRI using RECs could also benefit from the use of MEMSs for the active decoupling while additionally enabling reconfigurable REC geometries [36]. In this work, we focus on studying the feasibility of using a MEMS as an active decoupling element. This is an interesting question considering the very small size of the REC loop and therefore its resulting small electrical resistance with respect to that of
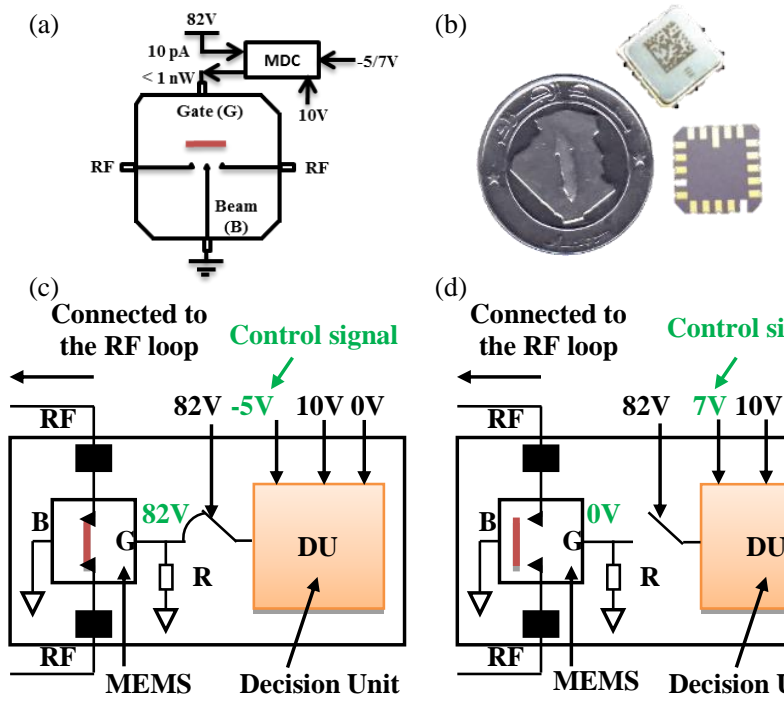

(d)

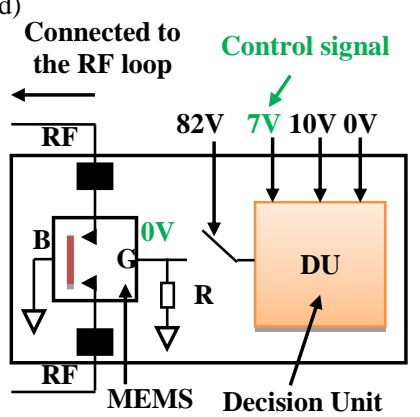

Fig. 1. Description of MEMS switch and its driver. (a) Scheme of the controllable RF MEMS switch developed by GE Healthcare Inc. (b) Photograph of the $1 \mathrm{~cm} \times 1 \mathrm{~cm}$ chip package of only the MEMS switch with 1 Algerian dinar coin. Second line shows two small diagrams of the MEMS and its driver circuit (MDC). The MDC consists of an electronic Decision Unit (DU) biased with $10 \mathrm{~V}$ and $0 \mathrm{~V}$ input voltages and allowed to compare a predetermined threshold voltage to the control signal. (c) If the DU receives a control signal of $-5 \mathrm{~V}$, an external $82 \mathrm{~V}$ (typical value) is delivered between the gate $(\mathrm{G})$ and beam (B) electrodes of the MEMS thereby closing the switch. (d) If the DU receives a control signal of $7 \mathrm{~V}$ (or $100 \mathrm{~mA}$ delivered by the MRI system), the external $82 \mathrm{~V}$ is blocked and cannot be delivered to the MEMS gate. In this case, $0 \mathrm{~V}$ is delivered between $\mathrm{G}$ and $\mathrm{B}$ thereby opening the switch. 


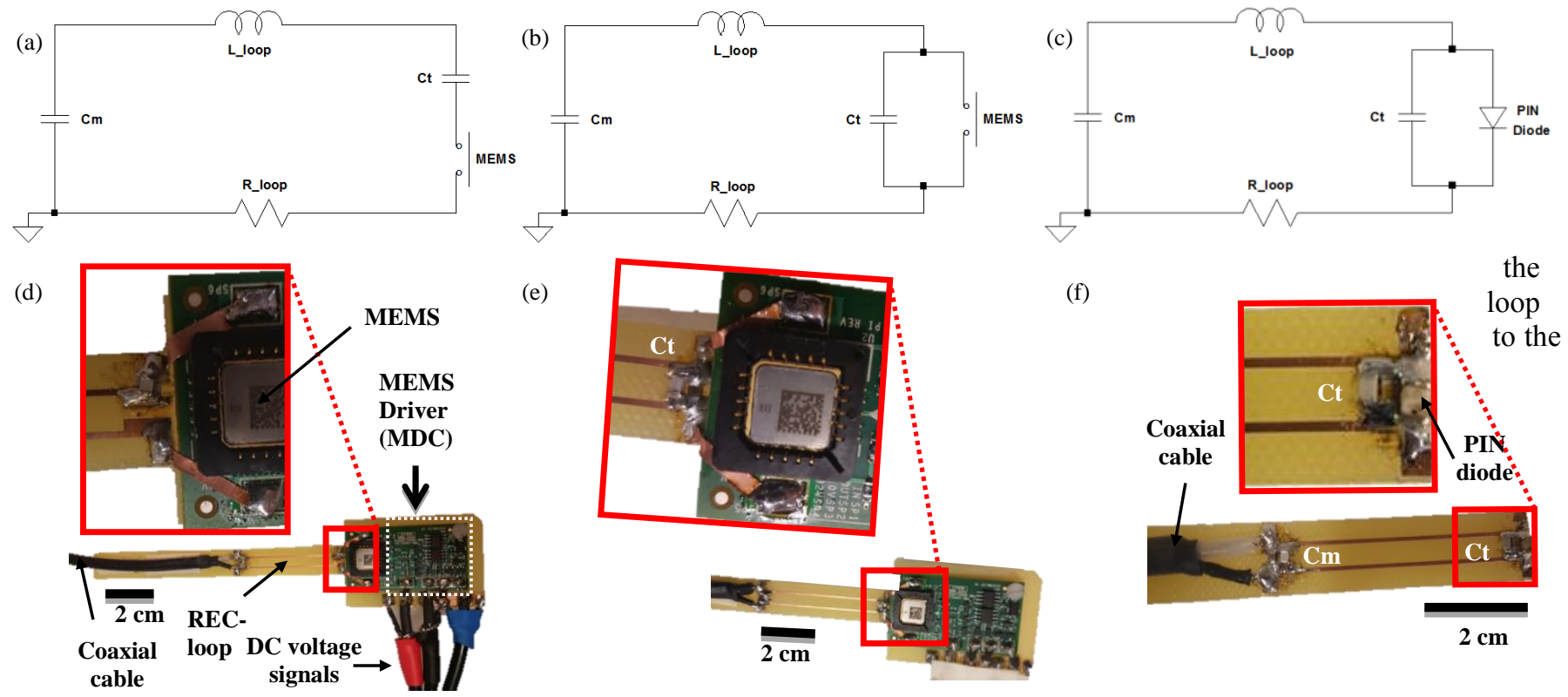

Fig. 2. Electrical schematics (first row) and the associated built prototypes (second row) of RECs using a controllable MEMS switch for active decoupling integrated $(\mathrm{a}, \mathrm{d})$ in series $(s$ MEMS REC) or $(\mathrm{b}, \mathrm{e})$ in parallel ( $p$ MEMS REC) to the loop or using $(\mathrm{c}, \mathrm{f})$ a PIN diode integrated in parallel to the loop $(p$ PIN REC). $\mathrm{Ct}$ and $\mathrm{Cm}$ are tuning and matching capacitors, respectively. R_loop and L_loop are the electrical resistance and inductance of the rectangular loop with $5.1 \mathrm{~mm}$ $\times 47 \mathrm{~mm} \times 0.8 \mathrm{~mm}$ of width, length and thickness dimensions, respectively. The coaxial cable is the RF output used to transmit the NMR signal.

the MEMS. Since the impact of MEMSs used in parallel to the loop (the MEMS is similar to either an additional capacitor or a short circuit shifting the REC resonance frequency) is not the same as the impact of the MEMS used in series with the loop (the MEMS is similar to either an additional parasitic resistance or an open circuit in the REC loop), we first tested the two configurations not only in terms of active decoupling performance but also in terms of an active image quality to detect the performance advantages and drawbacks of each configuration.

This manuscript presents the first step of our project. For this purpose, two RECs with active decoupling circuits using MEMS placed in series or parallel to the loop were assessed and compared with a reference REC using a conventional PIN diode. All RECs were characterized on experimental benches, in both coupled and decoupled states. Tuning, matching, quality factor and isolation (decoupling efficacy) were measured. Switching delays to couple or decouple the REC were also estimated. Finally, the three RECs were tested in imaging conditions on a 1.5-T MR system. SNR mean and distribution were measured to assess differences on the acquired image.

\section{MATERIALs AND Method}

\section{A. REC prototypes}

In this study, three copper RECs with identical rectangular loop geometries were mechanically etched on an FR4 epoxy substrate of 1.6-mm thickness (S63, LPKF Laser and Electronics, Germany). Each REC consisted of a single rectangular loop $(5.1-\mathrm{mm}$ width, 47-mm length and 35- $\mathrm{mm}$ copper track thickness). In order to operate with a 1.5-T MRI system, fixed capacitors (ATC, New York, USA and Temex Ceramics Exxelia, Pessac, France) were soldered in the proximal side to match $50 \Omega$ and in the distal position to tune working proton resonance frequency (63.87 MHz).

MEMS switches (MM7100, Menlo Microsystems, Inc., Irvine, CA, USA) were used in this study to couple or decouple the REC during endoluminal MRI. The MEMSs used are fast mechanical switches based on a mobile micro metallic actuator (Fig. 1-a) inside a chip of $1 \mathrm{~cm} \times 1 \mathrm{~cm}$ size (Fig. 1-b). The MEMS is open by default and to close it, sufficient voltage (in our case, the typical value was $82 \mathrm{~V}$ ) has to be applied between the gate $(\mathrm{G})$ and the beam $(B)$ of the MEMS. For our MRI application, since the MR system was not able to deliver these voltages, a driver circuit supplied the required voltage to open or close the MEMS so as to achieve the coupling/decoupling of the REC. As can be seen in the two schematics of the MEMS Driver Circuit (MDC) in Fig. 1-c,d, the MDC consists of an electronic decision unit (DU) and is equipped with four inputs. Three of them are used to receive continuously the required bias voltages: $0 \mathrm{~V}$ as ground, $10 \mathrm{~V}$ as bias voltage of the DU and $82 \mathrm{~V}$ to close the MEMS switch. The last input receives the control signal and directs it to the DU, which compares it to a predetermined threshold voltage leading to the delivery or blocking of the $82 \mathrm{~V}$ to the gate of the MEMS. Applying a $-5 \mathrm{~V}$ control signal to the DU delivers the $82 \mathrm{~V}$ to the MEMS gate $(\mathrm{G})$ and thus closes the MEMS switch (Fig. 1-c), while a 7-V voltage (which is equivalent to a current of $100 \mathrm{~mA}$ provided by the MRI system) leads to $0 \mathrm{~V}$ being applied between $G$ and $B$ and thus opens the switch (Fig. 1-d). More details about the MEMS and MDC information were provided in previous work [37].

To evaluate the MEMS performances for active decoupling of RECs, two different active decoupling REC configurations with MEMS in series ( $s$ MEMS REC) and MEMS in parallel ( $p$ MEMS REC) to the loop were built and assessed. In the $s$ MEMS REC configuration (Fig. 2a-d), the MEMS switch was integrated into the loop (in series with the tuning capacitor $\mathrm{Ct}$ ). During transmission, the MEMS must be open 
capacitor $\mathrm{Ct}$ ). During transmission, the MEMS must be open (REC is not resonating). In the $p$ MEMS REC (Fig. 2b-e), the switch was integrated in parallel to the tuning capacitor $\mathrm{Ct}$. During transmission, the MEMS must be closed to shift the resonance frequency and thus detune the REC. In addition, a third REC was built with a conventional decoupling circuit using a PIN diode component (DH 80055, Temex Ceramics, Pessac, France) placed at a distal position in parallel ( $p$ PIN REC) to the tuning capacitor (Fig. 2c-f). The PIN diode was controlled using a forward biased voltage of $3.8 \mathrm{~V}$ (which is equivalent to a current of approximately $100 \mathrm{~mA}$ provided by the MRI system). A summary of tuning and matching capacitor values used to build the different RECs is given in Table I.

TABLE I

TUNING AND MATCHING CAPACITOR VALUES USED

\begin{tabular}{llll}
\hline \hline RECs & MR switches & $\begin{array}{l}\text { Ct values } \\
(\mathrm{pF})\end{array}$ & $\begin{array}{l}\text { Cm values } \\
(\mathrm{pF})\end{array}$ \\
\hline$p$ MEMS & MEMS & 142.0 & 582.0 \\
$s$ MEMS & MEMS & 120.2 & 382.0 \\
$p$ PIN & PIN diode & 158.2 & 549.0 \\
\hline \hline
\end{tabular}

REC prototypes were characterized on two experimental measurement benches as well as by imaging using a phantom made of a cylindrical vessel $(90 \mathrm{~mm}$ outer diameter, $100 \mathrm{~mm}$ long) filled with a solution of $1.25 \mathrm{~g} \mathrm{NiSO} 4 \times 6 \mathrm{H}_{2} \mathrm{O}+5 \mathrm{~g}$ $\mathrm{NaCl}$ per liter of distilled water mimicking tissue losses and with a through-hole (11 mm inner diameter) allowing for the introduction of the REC loops (Fig. 3). For ease of use, three identical phantoms were built, one for each REC prototype.
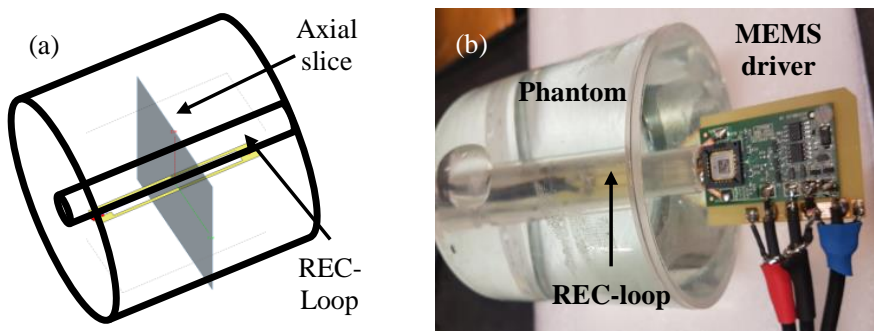

Fig. 3. (a) Drawing and (b) photograph of the cylindrical phantom used to load the REC for characterization as well as for imaging. The dimensions of the cylindrical phantom are $11 \mathrm{~mm}$ inner and $90 \mathrm{~mm}$ outer diameters and $100 \mathrm{~mm}$ length. It is filled with a solution of $1.25 \mathrm{~g} \mathrm{NiSO}_{4} \times 6 \mathrm{H}_{2} \mathrm{O}+5 \mathrm{~g} \mathrm{NaCl}$ per liter of distilled water mimicking tissue losses.

\section{B. Experimental bench characterization set-up}

Two complementary experimental electronic benches were used to assess the frequency and temporal responses of the different REC prototypes. Each REC was first connected to a Vector Network Analyzer (VNA) (Agilent Technologies Inc., E5071C, Santa Clara, CA, USA) to measure the reflection coefficient $S_{11}$ in both coupled and decoupled states (Fig. 4ab). In the coupled state, the reflection coefficient response $\left(\mathrm{S}_{11}\right)$ enabled tuning and matching of each REC at the Larmor resonance frequency $\left(\mathrm{F}_{0}\right)$ by choosing the adequate tuning and matching fixed capacitors. Thus, the quality factor (Q-value)
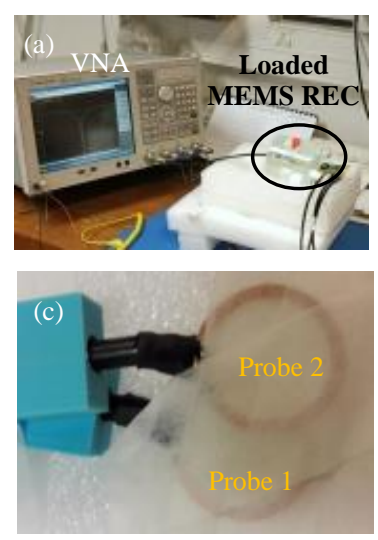
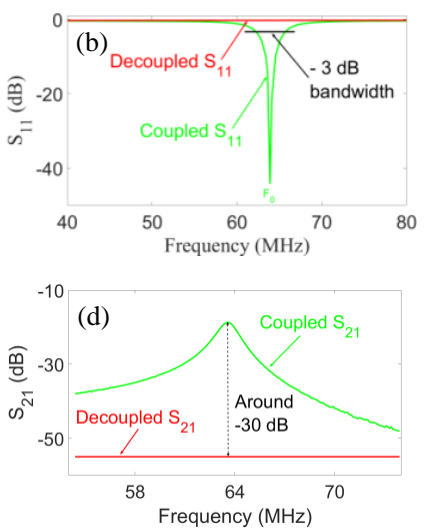

Fig. 4. First RF characterization set-up image (a) and the associated reflection coefficient $\left(\mathrm{S}_{11}\right)$ measurement method (b) by using a VNA. At coupling state (resonance frequency), amplitude at Larmor resonance frequency $\left(\mathrm{F}_{0}\right)$ and quality factor were derived from the $S_{11}$ response. At decoupling state, $S_{11}$ amplitude at $F_{0}$ was measured. (c) Decoupled dual loops position. (d) Second RF characterization set-up image and (e) the associated $S_{21}$ measurement method using the VNA.

of each REC was derived from $\mathrm{S}_{11}$ response at $-3 \mathrm{~dB}$ bandwidth [38]-[40].

To assess the decoupling efficacy with a capacitive method, the $S_{11}$ response in the decoupled state was first used. In parallel decoupling strategies ( $p$ MEMS and $p$ PIN RECs), decoupling is achieved by detuning the RECs (shifting of the resonance frequency) resulting in no resonance at $\mathrm{F}_{0}$, which is characterized by the associated $S_{11}$ value at $F_{0}$ close to $0 \mathrm{~dB}$. In the case of $s$ MEMS REC, the switch is opened for a completely open REC loop thereby achieving active decoupling with a similar result in $\mathrm{S}_{11}$ at $\mathrm{F}_{0}$.

To confirm the obtained results, RECs were tested again with a mutual-induction method [41] based on the $S_{21}$ measurements. Two homemade flux probes (Fig. 4-c) were connected to the two ports of a VNA and overlapped to be mutually decoupled in free space (parallel, adjacent and overlapping loops). The REC was then placed close to the two probes such that if it resonated at $\mathrm{F}_{0}$, a mutual coupling between the previously decoupled probes was created via the REC and specifically at its resonance frequency $F_{0}$. On the contrary, if the REC was decoupled then at $\mathrm{F}_{0}$ there was no longer a coupling between the probes and the $S_{21}$ measured would drop significantly (by at least $30 \mathrm{~dB}$ ). This is illustrated in Fig. 4-d. For both $S_{11}$ and $S_{21}$ measurements, coupled/decoupled states were achieved by driving the opening or closing of the MEMS switch as explained previously.

To assess the decoupling speed, switching delays from the coupled state to the decoupled state and vice versa were measured. A circular broadband copper loop (41 mm inner diameters) connected to a frequency generator (HAMEG Instruments HM 8134-3, Mainhausen, Germany) was used to generate an RF signal at $\mathrm{F}_{0}$. By inductive coupling with the REC, the induced RF signal was measured using a digital oscilloscope (LeCroy waveJet 314, Lake Mary, FL, USA). To estimate the switching delays, a periodic square electric signal generated by a Waveform Generator (WG) (Agilent Technologies Inc. 33220A, Santa Clara, CA, USA) was used as coupling/decoupling control signal $(-5 \mathrm{~V} / 7 \mathrm{~V}$ for MEMS 
on the MDC control input and $3.8 \mathrm{~V} /-10 \mathrm{~V}$ for the PIN diode) (Fig. 5-a). The coupling and decoupling delays were measured as the time between the DC square control signal and the RF response signal (Fig. 5-b).

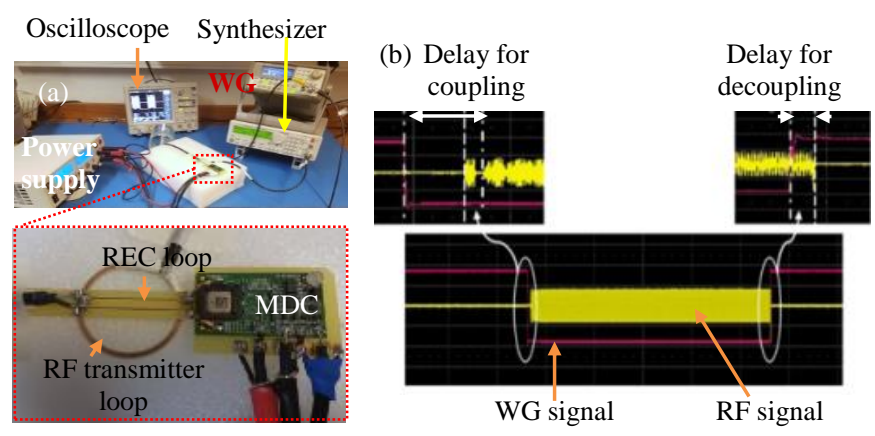

Fig. 5. Switching delays set-up image (a) and the temporal response measurements (b) of the MEMS Receiver Endoluminal Coil (REC). A frequency generator excites a circular copper loop to generate an RF magnetic field at the resonance frequency of the REC. The latter detects the RF signal while in coupled state but is unable to do so in the decoupled state. Coupling and decoupling are ensured by applying a square electrical signal from the WG. The received signal is observed on a digital oscilloscope. (b) Then, delays to couple or decouple the REC are estimated by measuring the delay between the applied control signal and the receive RF signal response.

\section{MRI set-up}

MRI experiments were performed on a clinical Optima MR450w 1.5-T MR scanner (General Electric, Inc. Trademark) (Fig. 6). The body coil was used as RF transmitter. Both the REC and phantom were placed within the scanner with their axes aligned with the $\mathrm{B}_{0}$ field in order to acquire $2 \mathrm{D}$ axial images. To interface the homemade REC to the MR system, a single-channel connector (A-plug) including a preamplifier (managed directly by the MRI system) with the appropriate configuration file was used. An RF circuit (RF/DC splitter) was placed between the RF channel of the A-plug connector and the REC in order to divert the DC control logic signal provided by the MR scanner. This was necessary because before starting a sequence, the MR system checks whether a coil is correctly plugged by verifying that a current can flow in the decoupling circuit. A decoy circuit was used in the case of MEMS RECs.

It should be noted that the DC control logic signal supplied by the MR scanner during the sequence does not always match the input control voltage required by the MEMS control circuit. Indeed, in the case of the $p$ MEMS REC design, the MR control logic (out of the MRI system) has to be inverted. Since the MR scanner we used was not configurable to perform this task, our solution was to use a waveform generator (WG). Thus, $p$ MEMS REC was controlled in this work only by using the WG ( $p$ MEMS $_{W G}$ REC), while the $s$ MEMS REC was controlled either directly by the MR system ( $s$ MEMS REC), as in the case of the $p$ PIN REC and also with WG $\left(s\right.$ MEMS $_{W G}$ REC) so as to be able to characterize the effect of using the WG. For both MEMS configurations using a WG, a control signal of $-5 \mathrm{~V} / 7 \mathrm{~V}$ was generated with the WG while a control signal of $-5 \mathrm{~V} / 100 \mathrm{~mA}$ was used with the

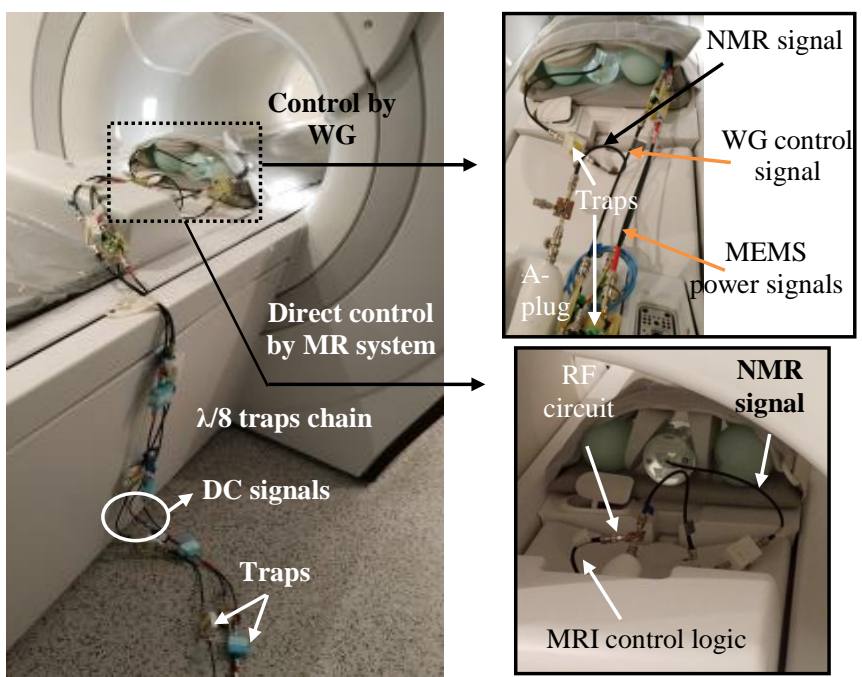

Fig. 6. Implementation on a 1.5-T MR system of the Receiver Endoluminal Coil (REC) using controlled MEMS switch. The body coil is used as an RF transmitter coil and the endoluminal coil as receiver. The RF circuit makes it possible to deviate the DC signal. The trigger by the WG makes it possible to ensure the DC control without the need to add an inverter circuit in the case of $p$ MEMS REC. The REC is connected to the MR scanner via a specific interfacing connector (A-plug) with an adequate file configuration to identify the REC. Since both REC and phantom have their axes (lengths) aligned with the $\mathrm{B}_{0}$ field, the 2D axial images are acquired using gradient echo (GRE) and spin echo (FSE) sequences.

A-plug of the MRI system. An external power supply (Electro-Automatik EA-PS 2384-03B, Viersen, Germany) was used to supply $10 \mathrm{~V}$ and $82 \mathrm{~V}$ (bias signals) to the MDC. Both the power supply and the WG were placed outside the Faraday cage, in the technical room. Thus, three DC lines using coaxial cables ( $4.7 \mathrm{~m}$ for each line) were used to transport the three DC signals (10 V, $82 \mathrm{~V}$ and control signals). Traps were added every eighth of the wavelength $(\lambda / 8)$ of each coaxial cable (11 traps for each DC line) to avoid RF-induced signal [42], [43], which would otherwise lead to not only strong image quality degradation but also damage to the device (MEMS or/and MDC). The same was done on the RF signal reception cable.

Multiple axial slices were acquired using gradient echo (GRE) and fast spin echo (FSE) sequences. Sequence acquisition parameters are summarized in Table II.

The $s$ MEMS, $p$ PIN and $p$ MEMS $_{W G}$ REC prototypes were compared in terms of mean SNR and SNR isocontours. Although the MR control logic can be used directly to control the $s$ MEMS REC, the WG and the associated input control voltage line (including traps) were also used to assess the effects of employing the WG on image quality $\left(s\right.$ MEMS $_{W G}$ REC).

Images were post-processed using Matlab software (Mathworks, Milwaukee, USA), and mean SNR and signal uniformity distributions were calculated. The SNR was determined as the ratio of the mean signal intensity calculated on the entire phantom image and the standard deviation of a rectangular ROI placed outside the phantom. The SNR distribution map (SNR isocontours) was calculated and drawn for each REC. Then, mean SNR values were calculated for 
TABLE II

ACquisition PaRameters USEd for Both Gradient Echo (GRE) AND SPIN Echo (FSE) SeQuences.

\begin{tabular}{|c|c|c|c|c|c|c|c|c|c|}
\hline Sequence & Orientation & TR/TE (ms) & $\mathrm{FA}\left({ }^{\circ}\right)$ & $\begin{array}{l}\text { FOV } \\
(\mathrm{mm} \times \mathrm{mm})\end{array}$ & $\begin{array}{l}\text { Slice } \\
\text { thickness } \\
(\mathrm{mm})\end{array}$ & $\begin{array}{l}\text { Spacing } \\
\text { slices } \\
(\mathrm{mm}) \\
\end{array}$ & Matrix size & $\begin{array}{l}\text { Receive } \\
\text { BW } \\
( \pm \mathrm{kHz}) \\
\end{array}$ & $\begin{array}{l}\text { Number } \\
\text { of slices }\end{array}$ \\
\hline GRE & Axial & $400 / 9$ & 80 & $100 \times 100$ & 2 & 0.5 & $256 \times 256$ & 11.9 & 13 \\
\hline FSE & Axial & $3100 / 14.1$ & $90 / 180$ & $100 \times 100$ & 2 & 0.2 & $256 \times 256$ & 15.6 & 13 \\
\hline
\end{tabular}

FSE acquisitions on concentric circles centered on the REC loop for radiuses between $11 \mathrm{~mm}$ and $22 \mathrm{~mm}$.

\section{Results}

The measured frequency and temporal response parameters for the three REC prototypes in coupled and decoupled states are summarized in Table III. In coupled state, reflection coefficient values $S_{11}$ at $F_{0}$ were approximately $-30 \mathrm{~dB}$ for all RECs. The loaded quality factor of the $s$ MEMS was significantly lower $(37 \%)$ than the $p$ MEMS REC, which in turn had a slightly higher Q-value that the $p$ PIN REC. Unloaded (data not reported) and loaded Q-values were almost similar for each individual REC.

In the decoupled state, measured reflection coefficient values $\mathrm{S}_{11}$ at $\mathrm{F}_{0}$ were measured to be almost $0 \mathrm{~dB}$ (no resonance) for all RECs. Resonance frequencies of $p$ MEMS and $p$ PIN RECs were shifted from $63.95 \mathrm{MHz}$ to $20.47 \mathrm{MHz}$ and $29.3 \mathrm{MHz}$, respectively. In the case of the $s$ MEMS REC, the loop is completely open and thus the REC was not resonating at all. The mutual decoupling of the two loops at $F_{0}$ was also shown with a drop in $S_{21}$ measurements of more than $30 \mathrm{~dB}$ for all RECs.

Switching delays to both coupled and decoupled states were less than $0.7 \mu$ s for the $p$ PIN REC. Regarding both MEMS RECs, coupling delays were equal to $7.5 \mu \mathrm{s}$ and $2.1 \mu$ s for $s$ MEMS and $p$ MEMS, respectively, while decoupling delays were equal to $1.8 \mu \mathrm{s}$ and $9.8 \mu \mathrm{s}$ for $s$ MEMS and $p$ MEMS, respectively.

TABLE III

MEASUREd FrEQUENCY AND TEMPORAL RESPONSE PARAMETERS FOR THE THREE COIL PROTOTYPES WITH COUPLING AND DECOUPLING STATES.

\begin{tabular}{lllll}
\hline \hline \multirow{2}{*}{ States } & Measured & $p$ PIN & $s$ MEMS & $p$ MEMS \\
\hline \multirow{4}{*}{ Coupled } & $\mathrm{F}_{0}(\mathrm{MHz})$ & 63.95 & 63.92 & 63.95 \\
& $\mathrm{~S}_{11}$ at $\mathrm{F}_{0}(\mathrm{~dB})$ & -28.70 & -36.40 & -29.20 \\
& $\mathrm{~S}_{21}$ at $\mathrm{F}_{0}(\mathrm{~dB})$ & -18.45 & -26.20 & -20.30 \\
& $\mathrm{Q}_{\text {Loaded }}$ & 62.50 & 41.20 & 65.10 \\
\hline \multirow{2}{*}{ Decoupled } & $\mathrm{S}_{11}$ at $\mathrm{F}_{0}(\mathrm{~dB})$ & -0.28 & -0.09 & -0.10 \\
& $\mathrm{~S}_{21}$ at $\mathrm{F}_{0}(\mathrm{~dB})$ & -48.10 & -56.10 & -55.00 \\
\hline \multirow{5}{*}{} & Delays to couple $(\mu \mathrm{s})$ & 0.70 & 7.50 & 2.08 \\
& Delays to decouple $(\mu \mathrm{s})$ & 0.25 & 1.84 & 9.80 \\
\hline \hline
\end{tabular}

For imaging experiments, the presence of traps on the DC control line and DC power supply lines of the MDC was mandatory in order to have an unaltered DC bias and stable power supply during the scan. Indeed, in the absence of traps, RF signals induced by the transmitter body coil were superimposed onto the DC control signal (up to $20 \mathrm{~V}$ peak to peak, depending on the sequence used) and could

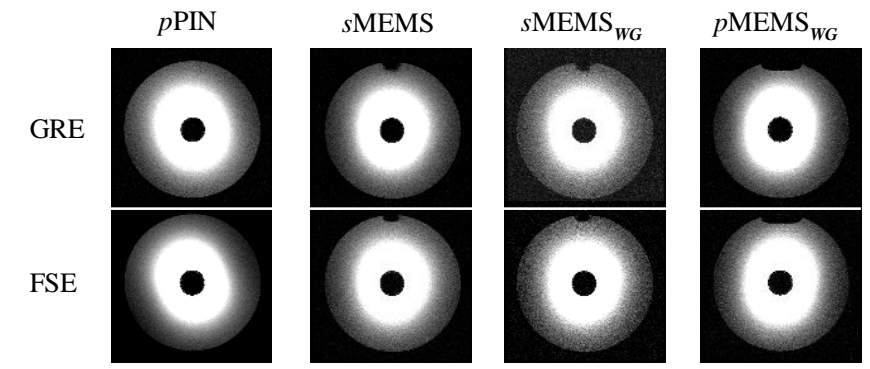

Fig. 7. Representative 2D axial MR images acquired with GRE and FSE sequences for the different RECs prototypes (PIN and MEMS) and displayed using a narrow signal intensity window.

inappropriately couple or decouple the REC. In that case, the acquired image is extremely noisy and moreover, could lead to damaging the MDC board and/or the MEMS switch. This occurs when the number of traps is inadequate.

From a qualitative point of view, Fig. 7 shows that the obtained images display similar elliptical image sensitivity patterns and no signal saturation even close to the REC. No artifacts due to active decoupling failure were observed on the images when using GRE or FSE. Small holes were observed on each image (anterior part of the phantom) obtained by both MEMS RECs.

Mean SNR values calculated for FSE acquisitions on concentric circles centered on the REC loop for radiuses between $11 \mathrm{~mm}$ and $22 \mathrm{~mm}$ showed that for all radiuses, SNR decreased in the following order:

$p$ PIN > $s$ MEMS $>p$ MEMS $_{W G}>s$ MEMS $_{W G}$. The SNR isocontours calculated for all experimental configurations and prototypes using FSE sequences are displayed in Fig. 8. Figure 8-a enables an easy comparison of the image quality of $s$ MEMS, $p$ MEMS $_{W G}$ and $p$ PIN RECs together; Fig. 8-b compares $s$ MEMS $_{W G}$, with $p$ MEMS $_{W G}$ (performance of $s$ MEMS and $p$ MEMS in the same control conditions), while a comparison between $s$ MEMS and $s$ MEMS $_{W G}$ (WG control technique effect) is presented in Fig. 8-c.

\section{DISCUSSION}

In this work, we evaluated the serial and parallel active decoupling of MEMS REC performances in terms of switching delays, quality factor, decoupling isolation (efficiency), delays (speed) and image SNR.

Opening and closing delays of the MEMS itself are $1 \mu \mathrm{s}$ and $4 \mu \mathrm{s}$, respectively, which largely explains the decoupling and coupling delay differences of the same MEMS REC.

Opening of the MEMS leads to coupling of the $p$ MEMS REC and decoupling of the $s$ MEMS REC, which thus display almost similar delays of $2.1 \mu \mathrm{s}$ and $1.8 \mu \mathrm{s}$, respectiv 

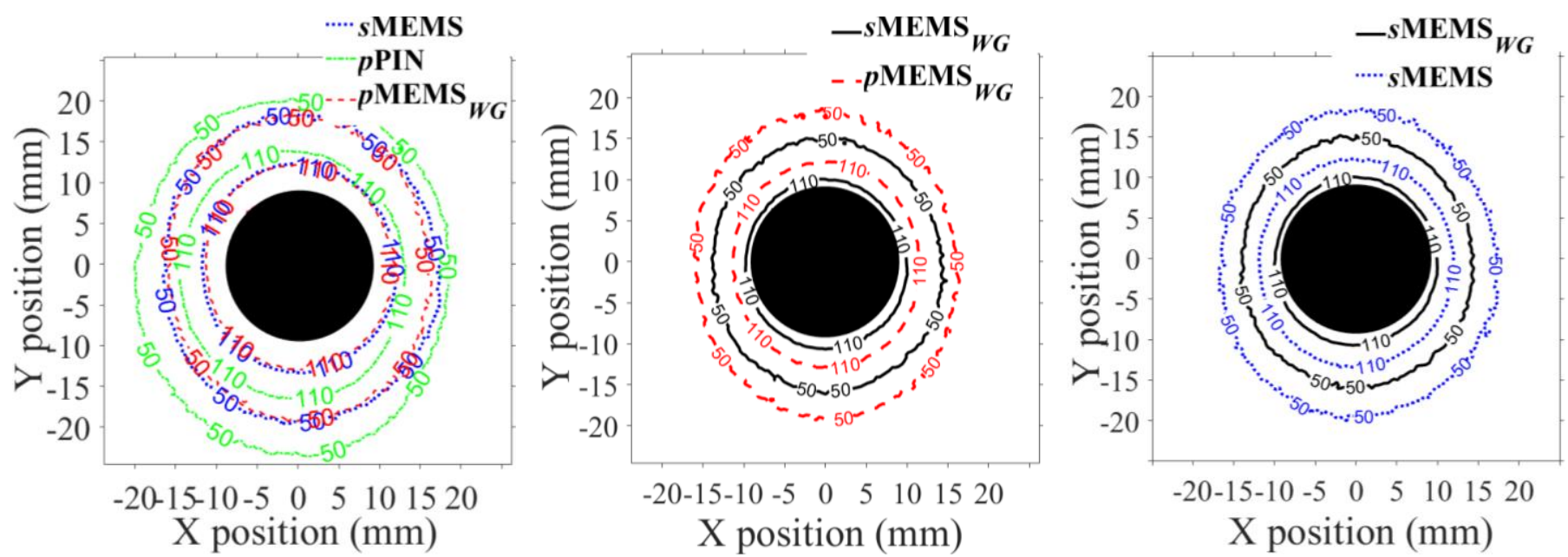

Fig. 8. Signal-to-Noise Ratio (SNR) distributions based on iso-contour lines calculated and drawn on axial FSE images acquired by using the different REC prototypes. Numbers on each iso-contour line represent the calculated SNR value obtained with the associated REC. (a) Comparison between $s$ MEMS,

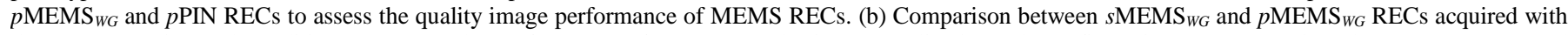
the same experimental conditions to assess the performance of MEMS on the image quality in each configuration. (c) Comparison between $s$ MEMS and $s \mathrm{MEMS}_{W G}$ RECs to evaluate the effect of the use of the waveform generator on the acquired images.

Closing of the MEMS switch instead leads to decoupling of the $p$ MEMS REC and coupling of the $s$ MEMS REC, which in this case display delays of $9.8 \mu$ s and $7.5 \mu$ s, respectively. The slight difference in delay $(2.3 \mu \mathrm{s})$ in the last case is probably due to the specific operation technique of each configuration (by closing the MEMS, the REC loop is closed in coupled state in the case of the $s$ MEMS REC while in the case of the $p$ MEMS REC, the resonance frequency is shifted by shortcircuiting the tuning capacitor). With switching delays between $0.25 \mu$ s and $0.7 \mu$ s, the reference $p$ PIN REC switches faster than the MEMS-based REC for coupled and decoupled states. These relatively longer delays are due to the time necessary to move the switch mechanically and to the switching delays of its driver circuit [33]. The increased delays by adding such driver circuit is also demonstrated in a recent work [44] using a PIN diode driver for high-power pulses where the rise-time is close to $1 \mu \mathrm{s}$ and the fall-time increases to $7.4 \mu \mathrm{s}$, which are comparable to the results obtained with controlled MEMSs. Delays are nevertheless of the same order as optical-based decoupling circuits with $13.6 \mu \mathrm{s}$ and $1.7 \mu \mathrm{s}$ for tuning and detuning, respectively [23], which proved to be effective. In any case, MEMS switching delays are still small and compliant with most MR clinical applications where the RF pulse duration of imaging sequences as well as signal readout time [33] are of the order of milliseconds. The only case that would be problematic in our application is that of ultra-short echo times (UTE) in the range of a few microseconds.

An equivalent REC devoid of any decoupling circuit was built and characterized and it has a $\mathrm{Q}$-value of 66. Among the REC loop, the $p$ MEMS REC had the highest Q-value ( 65). It is comparable to the $p$ PIN REC ( 62), with the lowest value being for the $s$ MEMS REC ( 41). This is due to the MEMS characteristics and its location on the REC loop. In the case of the $p$ MEMS REC in the coupled (reception) state, the MEMS is open. In this state, it can be considered as an additional capacitor of only about $1.5 \mathrm{pF}$ placed in distal position in parallel to the tuning capacitor of the REC. Because it is a mechanically open switch, it has a high isolation resistance of approximately $10 \mathrm{M} \Omega$ Thus, the MEMS does not impact the real part of the REC impedance, which affects the quality factor minimally. Rescia et. al. [24] also demonstrated on a 4$\mathrm{cm}$ surface coil at $400 \mathrm{MHz}(9.4 \mathrm{~T})$ that the MEMS, placed in parallel to the loop, had almost no effect on the quality factor compared with other electrical components such as PIN diodes. In the case of the REC used, the PIN diode had a very moderate effect on the Q-factor. For the $s$ MEMS REC, the switch is closed in the reception phase. It can then be considered as a significant $0.5 \Omega$ to $1 \Omega$ parasitic resistance [31], [37] in the loop that should be compared with the loop size and its equivalent electrical resistance. This explains the experimental 0.62 ratio of Q-values between the $s$ MEMS REC and a REC without any decoupling circuit. This ratio is comparable to the 0.59 theoretical Q-ratio calculations based on resistive losses [45].

Regarding decoupling efficacy, $\mathrm{S}_{11}$ responses of around $0 \mathrm{~dB}$ demonstrate an efficient active decoupling of all RECs especially for $s$ MEMS REC with an open loop. For all RECs, the $S_{21}$ results confirm the decoupling efficacy with a drop of more than $30 \mathrm{~dB}$ in $\mathrm{S}_{21}$ measurements at $\mathrm{F}_{0}$. This was confirmed on MR images where no signal artifacts were detected.

Signal intensity distribution, SNR isocontour and mean SNR values of MR images obtained with $s$ MEMS, $p$ PIN and $p \mathrm{MEMS}_{W G}$ RECs confirm that MEMSs are relevant systems for achieving active decoupling. In our experiments, the $p$ PIN REC always provided the highest SNR. This was expected compared with the sMEMS REC, which had the lowest Qvalue owing to the presence of an additional parasitic resistance in the receive phase. In addition, despite the $s$ MEMS decoupling signal being generated by the MR scanner through the A-plug, the external power supply was still used (to provide $10 \mathrm{~V}$ and $82 \mathrm{~V}$ bias voltages) and thus associated coaxial cables and traps were also used, which led to an additional noise on the images. 

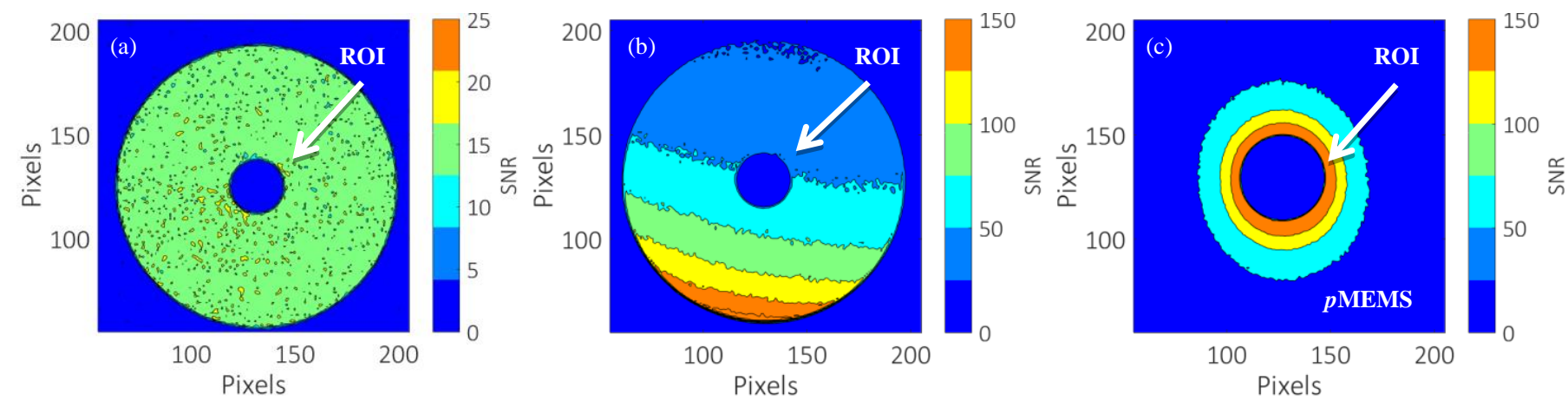

Fig. 9. SNR maps obtained with (a) the whole-body coil, (b) an external commercial array coil and (c) the $p$ MEMS REC. The $p$ MEMS REC provides an SNR higher than both the WBC (gain of 10) and the array coil. The latter displays high SNR only on the anterior surface of the phantom, (which is not our region of interest) with a rapid decrease when moving away from it. Since our region of interest is the internal surface, the array coil is not a convenient solution for our imaging target.

In the case of the $p \mathrm{MEMS}_{W G}$ REC, SNR values are lower than those of the $p$ PIN REC, which is not in agreement with the RF bench characterization results in terms of Q-value that was the highest for the $p$ MEMS REC and thus we expected a better or at least similar SNR between $p$ MEMS and $p$ PIN RECs. As mentioned above, the use of an external power supply for the MDC of all MEMS-based RECs is the first factor affecting the measured SNR values negatively. In the case of the $p \mathrm{MEMS}_{W G} \mathrm{REC}$, there is an additional source of noise originating from the external WG used to obtain the required coupling and decoupling signals (the MR system used could not handle it directly).

$s \mathrm{MEMS}_{W G}$ and $p \mathrm{MEMS}_{W G}$ REC experiments based on the same control condition (waveform generator) shown in Fig. 8b demonstrate that the $p \mathrm{MEMS}_{W G}$ REC presents a higher SNR (about $45 \%$ ) than the $s \mathrm{MEMS}_{W G}$ REC, which means that the $p$ MEMS REC (without WG control) can be estimated to yield an SNR higher $(45 \%)$ than the sMEMS REC, in agreement with the obtained Q-values.

In order to characterize this added noise due to the external WG, images were also acquired using the sMEMS REC with the decoupling signal coming from the external wave generator $\left(s \mathrm{MEMS}_{W G} \mathrm{REC}\right)$. The resulting images (Fig. 7 columns 2 and 3) and SNR isocontours (figures 8-c) demonstrate that the use of the external WG control leads to an SNR reduction of approximately $35 \%$, which can also be used to extrapolate SNR values that could be obtained with a $p$ MEMS REC (without the use of the external WG). The $p \mathrm{MEMS}_{W G}$ performance compared with the $p \mathrm{PIN}$ performance is hence significantly penalized by this step, which was mandatory because of the use of an MR450w system. Performing the experiments on a new GE MR scanner would ease the use of MEMS as a decoupling component thanks to the native integration of $82-\mathrm{V}$ and $10-\mathrm{V}$ delivery voltages as well as DC bias delivery for active decoupling in logic suited for all MEMS configurations. Such "on-board" voltage sources would then remove the additional noise from the mandatory setup described.

It should be noted that three separate but identical (size and solution) phantoms were used for the PIN, sMEMS and $p$ MEMS experiments. The small holes that can be seen on the
MEMS images correspond to air bubbles (see also Fig. 3-b) in each MEMS phantom due to the cylindrical vessel not being completely air-tight and not due to the MEMSs themselves. Coincidentally, the PIN phantom cylindrical vessel was almost completely air-tight and did not have air bubbles; thus, no hole was observed on the MR images obtained with the $p$ PIN REC.

In addition, SNR values in the ROI of the $p$ MEMS REC are much higher (factor of approximately 10) than those obtained with the Whole Body Coil (WBC) or with a commercial external array coil (GEM Flex Coil 16-S Array, 1.5T Receive Only, NeoCoil, USA) as shown in Fig. 9. It should be noted that the array coil displays its maximum SNR on the external surface of the phantom, which is not our ROI, and the SNR decreases rapidly when moving away from the surface so that in the ROI, its SNR is much lower than that achieved with our REC.

\section{CONCLUSION}

In conclusion, the $p \mathrm{MEMS}_{W G}$ configuration (based on an external control) presented here does not yet provide significant advantages over the $p$ PIN configuration (Fig. 8-a) mainly because of the external equipment required (WG, DC bias voltages, traps and coaxial cables). As perspectives, it is expected that the $p$ MEMS REC decoupling solution should present higher SNRs than the conventional $p$ PIN configuration, since the measured Q-values suggest that removing the external control and using an MR-compatible control method for the $p$ MEMS REC would yield higher SNRs than those achieved with the $p$ PIN REC. An interesting perspective for the use of MEMS switch resides in serial configuration (in series with the REC loop). Although the SNR is reduced, the decoupling remains efficient and MEMS could be used not only for active decoupling but also to allow for REC loop geometry reconfiguration. This could be particularly relevant in the case of colon wall examinations where REC-loop orientation with respect to the main magnetic field changes with location, leading to MR signal distribution changes. We believe that a combination of both $s$ MEMS and $p$ MEMS configurations in the design of new reconfigurable RECs may offer interesting designs and open the way for novel applications. 


\section{ACKNOWLEDGMENT}

The authors would like to thank Guido Kudielka, Julie Poujol, and Miguel Navarro from GE Healthcare for their help on MRI interfacing and configuration.

\section{REFERENCES}

[1] J. Ferlay et al., "Estimating the global cancer incidence and mortality in 2018: GLOBOCAN sources and methods," Int. J. Cancer, vol. 144, no. 8, pp. 1941-1953, 2019, doi: 10.1002/ijc.31937.

[2] C. Joachim et al., "Trends in colorectal cancer in the Caribbean: A population-based study in Martinique, 1982-2011," Rev. Epidemiol. Sante Publique, vol. 65, no. 3, pp. 181-188, 2017.

[3] X. V. Qadir, M. Clyne, T. K. Lam, M. J. Khoury, and S. D. Schully, "Trends in published meta-analyses in cancer research, 2008-2013," Cancer Causes Control, vol. 28, no. 1, pp. 5-12, 2017.

[4] L. A. Torre, F. Bray, R. L. Siegel, J. Ferlay, J. Lortet-Tieulent, and A. Jemal, "Global cancer statistics, 2012," CA. Cancer J. Clin., vol. 65, no. 2, pp. 87-108, 2015

[5] A.-M. Bouvier, "Epidémiologie descriptive du cancer colorectal en France," BEH Thématique, vol. 13, 2009.

[6] P. B. Roemer, W. A. Edelstein, C. E. Hayes, S. P. Souza, and O. M. Mueller, "The NMR phased array," Magn. Reson. Med., vol. 16, no. 2, pp. 192-225, Nov. 1990, doi: 10.1002/mrm.1910160203.

[7] A. Perrier et al., "Design of a Four-Channel Surface Receiver Coil Array Without Preamplifiers for the Decoupling Between Elements: Validation for High-Resolution Rat Knee MR Imaging," IEEE Sens. J., vol. 13, no. 6, pp. 2450-2458, Jun. 2013, doi: 10.1109/JSEN.2013.2254894.

[8] A. Perrier et al., "Design of a Two-Channel NMR Coil Using an Impedance Transformation Approach," IEEE Sens. J., vol. 12, no. 6, pp. 1801-1808, Jun. 2012, doi: 10.1109/JSEN.2011.2178237.

[9] M. Armenean, O. Beuf, F. Pilleul, and H. Saint-Jalmes, "Optimization of endoluminal loop radiofrequency coils for gastrointestinal wall MR imaging," IEEE Sens. J., vol. 4, no. 1, pp. 57-64, Feb. 2004, doi: 10.1109/JSEN.2003.820334.

[10] D. J. Gilderdale et al., "Design and use of internal receiver coils for magnetic resonance imaging.," Br. J. Radiol., vol. 72, no. 864, pp. 1141-1151, 1999.

[11] E. Atalar et al., "High resolution intravascular MRI and MRS by using a catheter receiver coil," Magn. Reson. Med., vol. 36, no. 4, pp. 596605, Oct. 1996, doi: 10.1002/mrm.1910360415.

[12] G. C. Hurst, J. Hua, J. L. Duerk, and A. M. Cohen, "Intravascular (catheter) NMR receiver probe: Preliminary design analysis and application to canine iliofemoral imaging," Magn. Reson. Med., vol. 24, no. 2, pp. 343-357, 1992, doi: 10.1002/mrm.1910240215.

[13] C. E. Hayes and L. Axel, "Noise performance of surface coils for magnetic resonance imaging at $1.5 \mathrm{~T}, "$ Med. Phys., vol. 12, no. 5, pp. 604-607, Oct. 1985, doi: 10.1118/1.595682.

[14] O. Beuf, F. Pilleul, M. Armenean, G. Hadour, and H. Saint-Jalmes, "In vivo colon wall imaging using endoluminal coils: Feasibility study on rabbits," J. Magn. Reson. Imaging JMRI, vol. 20, pp. 90-6, Jul. 2004, doi: 10.1002/jmri.20059.

[15] H. Dorez et al., "Endoluminal high-resolution MR imaging protocol for colon walls analysis in a mouse model of colitis," Magn. Reson. Mater. Phys. Biol. Med., vol. 29, no. 4, pp. 657-669, Aug. 2016, doi: 10.1007/s10334-016-0539-2.

[16] H. Dorez et al., "In vivo MRS for the assessment of mouse colon using a dedicated endorectal coil: initial findings," NMR Biomed., vol. 30, no. 12, p. e3794, 2017.

[17] G. Giovannetti, V. Hartwig, L. Landini, and M. F. Santarelli, "LowField MR Coils: Comparison between Strip and Wire Conductors," Appl. Magn. Reson., vol. 39, no. 4, pp. 391-399, Dec. 2010, doi: 10.1007/s00723-010-0173-5.

[18] W. A. Edelstein, C. J. Hardy, and O. M. Mueller, "Electronic decoupling of surface-coil receivers for NMR imaging and spectroscopy," J. Magn. Reson. 1969, vol. 67, no. 1, pp. 156-161, Mar. 1986, doi: 10.1016/0022-2364(86)90421-X.

[19] A. Reykowski, "Using memristor devices as switches for MRI RF coils," US9417299B2, Aug. 16, 2016.

[20] E. Wong, Q. Zhang, J. Duerk, J. Lewin, and M. Wendt, "An optical system for wireless detuning of parallel resonant circuits," J. Magn.
Reson. Imaging JMRI, vol. 12, pp. 632-8, Nov. 2000, doi: 10.1002/1522-2586(200010)12:4<632::AID-JMRI17>3.0.CO;2-J.

[21] S. Weiss et al., "In vivo safe catheter visualization and slice tracking using an optically detunable resonant marker," Magn. Reson. Med., vol. 52, no. 4, pp. 860-868, 2004, doi: 10.1002/mrm.20214.

[22] H. Eggers, S. Weiss, P. Boernert, and P. Boesiger, "Image-based tracking of optically detunable parallel resonant circuits," Magn. Reson. Med., vol. 49, no. 6, pp. 1163-1174, 2003, doi: $10.1002 / \mathrm{mrm} .10459$.

[23] I. Saniour et al., "Active optical-based detuning circuit for receiver endoluminal coil," Biomed. Phys. Eng. Express, vol. 3, no. 2, p. 025002, 2017, doi: 10.1088/2057-1976/aa5db0.

[24] S. Rescia and S. Smith, "RECEIVE-ONLY SURFACE COIL "WITH IMPROVED DETUNTNG FOR PRE-CLINICAL MR1 STUDIES," Brookhaven National Laboratory (BNL), 2013.

[25] P.-J. Nacher, S. Kumaragamage, G. Tastevin, and C. P. Bidinosti, “A fast MOSFET rf switch for low-field NMR and MRI," J. Magn. Reson., vol. 310, p. 106638, Jan. 2020, doi: 10.1016/j.jmr.2019.106638.

[26] R. Ayde et al., "Potentialities of an Electro-Optic Crystal Fed by Nuclear Magnetic Resonant Coil for Remote and Low-Invasive Magnetic Field Characterization," IEEE Sens. J., vol. 13, no. 4, pp. 1274-1280, Apr. 2013, doi: 10.1109/JSEN.2012.2230623.

[27] H. Fujita, T. Zheng, X. Yang, M. J. Finnerty, and S. Handa, "RF Surface Receive Array Coils: The Art of an LC Circuit," J. Magn. Reson. Imaging, vol. 38, no. 1, pp. 12-25, 2013, doi: 10.1002/jmri.24159.

[28] V. Taracila, K. Shet, and F. Robb, "Preamp decoupling-eigenvalue solution approach," channels, vol. 1, p. 1, 2008.

[29] C. C. Guclu, E. Boskamp, T. Zheng, R. Becerra, and L. Blawat, "A method for preamplifier-decoupling improvement in quadrature phased-array coils," J. Magn. Reson. Imaging, vol. 19, no. 2, pp. 255 258, 2004, doi: 10.1002/jmri.10449.

[30] D. Spence and M. Aimi, "Custom MEMS switch for MR surface coil decoupling," in Proceedings of the 23rd Annual Meeting of ISMRM, Toronto, Canada, 2015, p. 0704.

[31] S. B. Bulumulla, K. J. Park, E. Fiveland, J. Iannotti, and F. Robb, "MEMS switch integrated radio frequency coils and arrays for magnetic resonance imaging," Rev. Sci. Instrum., vol. 88, no. 2, p. 025003, Feb. 2017, doi: 10.1063/1.4975181.

[32] F. Maggiorelli et al., "Double-Tuned Surface 1H-23Na Radio Frequency Coils at 7 T: Comparison of Three Decoupling Methods," Appl. Magn. Reson., Feb. 2019, doi: 10.1007/s00723-019-01116-w.

[33] A. Maunder, M. Rao, F. Robb, and J. M. Wild, "Comparison of MEMS switches and PIN diodes for switched dual tuned RF coils," Magn. Reson. Med., vol. 80, no. 4, pp. 1746-1753, 2018, doi: 10.1002/mrm.27156.

[34] D. Darnell, Y. Ma, H. Wang, F. Robb, A. W. Song, and T.-K. Truong, "Adaptive integrated parallel reception, excitation, and shimming (iPRES-A) with microelectromechanical systems switches," Magn. Reson. Med., vol. 80, no. 1, pp. 371-379, 2018, doi: 10.1002/mrm.27007.

[35] K. Byron, S. A. Winkler, F. Robb, S. Vasanawala, J. Pauly, and G. Scott, "An MRI Compatible RF MEMs Controlled Wireless Power Transfer System," IEEE Trans. Microw. Theory Tech., vol. 67, no. 5, pp. 1717-1726, May 2019, doi: 10.1109/TMTT.2019.2902554.

[36] H. Raki, K. Tse-Ve-Koon, H. Souchay, F. Robb, S. Lambert, and O. Beuf, "Design of a reconfigurable endoluminal coil using MEMS switches," in 27th Annual meeting of the ISMRM, Montréal, Canada, May 2019, Accessed: Sep. 17, 2019. [Online]. Available: https://hal.archives-ouvertes.fr/hal-02266838.

[37] C. Keimel, G. Claydon, B. Li, J. N. Park, and M. E. Valdes, "Microelectromechanical-Systems-Based Switches for Power Applications," IEEE Trans. Ind. Appl., vol. 48, no. 4, pp. 1163-1169, Jul. 2012, doi: 10.1109/TIA.2012.2199949.

[38] D. Kajfez, "Q factor measurements, analog and digital," Univ. Miss. Dept Electr. Eng. Www Ee Olemiss Eduldarkorfqmeas2b Pdf, 1999.

[39] W. P. Wheless and D. Kajfez, "Experimental Characterization of Multimode Microwave Resonators Using Automated Network Analyzer," IEEE Trans. Microw. Theory Tech., vol. 35, no. 12, pp. 1263-1270, Dec. 1987, doi: 10.1109/TMTT.1987.1133846.

[40] D. Kajfez and E. J. Hwan, "Q-Factor Measurement with Network Analyzer," IEEE Trans. Microw. Theory Tech., vol. 32, no. 7, pp. 666-670, Jul. 1984, doi: 10.1109/TMTT.1984.1132751. 
[41] M. A. Griswold, "Characterization of Multichannel Coil Arrays on the Benchtop," in eMagRes, American Cancer Society, 2012.

[42] B. M. Schaller, A. W. Magill, and R. Gruetter, "Common modes and cable traps," in Proc. Intl. Soc. Mag. Reson. Med, 2011, vol. 19, p. 4660.

[43] D. M. Peterson, B. L. Beck, G. R. Duensing, and J. R. Fitzsimmons, "Common mode signal rejection methods for MRI: Reduction of cable shield currents for high static magnetic field systems," Concepts Magn. Reson. Part B Magn. Reson. Eng., vol. 19B, no. 1, pp. 1-8, 2003, doi: $10.1002 / \mathrm{cmr}$. b.10090.

[44] E. Der, V. Volotovskyy, H. Sun, B. Tomanek, and J. C. Sharp, "Design of a high power PIN-diode controlled switchable RF transmit array for TRASE RF imaging," Concepts Magn. Reson. Part B Magn. Reson. Eng., vol. 48B, no. 1, Feb. 2018, doi: 10.1002/cmr.b.21365.

[45] G. Giovannetti, V. Hartwig, L. Landini, and M. F. Santarelli, "Sample-Induced Resistance Estimation in Magnetic Resonance Experiments: Simulation and Comparison of Two Methods," Appl. Magn. Reson., vol. 40, no. 3, pp. 351-361, May 2011, doi: 10.1007/s00723-011-0210-z.

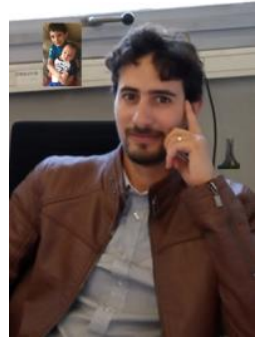

Hamza Raki was born in Beni Slimane, Algeria in 1987. He received his degree in Electronics and Instrumentation Engineering from USTHB (University of Sciences Technologies of Houari Boumediene), Algiers, Algeria in 2011. He has attained several master's degrees in MicroElectronics and Micro-Systems (University of Saad Dahlab, Blida, Algeria in 2013), Embedded Electronics (University of Loraine, Nancy, France in 2014), and Design of Integrated Devices and Circuits (University of Lyon 1, Lyon, France in 2016). He is preparing for a $\mathrm{PhD}$ degree in physics (clinical research and technological innovation). His current research focuses on the design and development of MEMS reconfigurable MRI endoluminal RF coils intended for colon wall imaging.

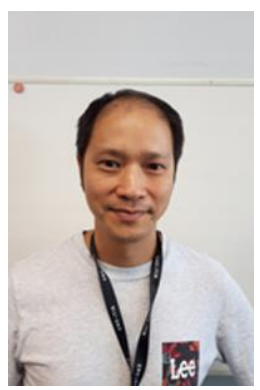

Kevin Tse Ve Koon was born in Mauritius in 1979. $\mathrm{He}$ received his $\mathrm{PhD}$ in physics in 2007 from the University of Burgundy, France. Thereafter he joined the University of Rennes for a postdoctoral fellowship in cardiovascular modeling and imaging and later joined the MR and optics team of CREATIS in 2010. His current research focuses on MR imaging, radiofrequency coils, MR elastography, and pulse designs through optimal control.

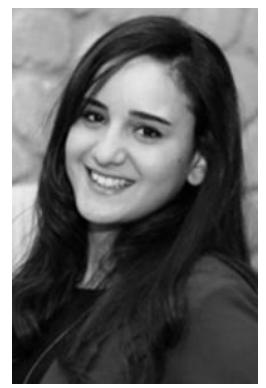

Isabelle Saniour was born in Mina, Lebanon in 1991. She received her master's degree in Sensors, Measurement, and Instrumentation from Pierre and Marie Curie University in partnership with ESPCI, Paris, France in 2013. She received a PhD degree in physics from Lyon 1 University in 2017. From 2018 to 2019, she was involved in research on superconducting radiofrequency coils at the University of Paris-Sud in Orsay, France. Her main research interests include the development of radiofrequency coils and hardware and the assessment of SAR for MR engineering applications.

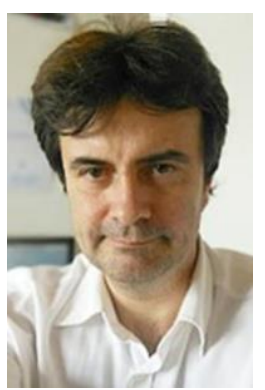

Henri Souchay graduated in engineering at Ecole Centrale Paris in 1994, and joined GE Healthcare in 1999 after attaining his PhD in Physics. After holding technical and leadership positions in image chain design engineering, in 2015 Henri moved to lead the clinical research team of GE Healthcare in France, and he now manages a broad portfolio of research activities with prominent institutions and researchers, while developing the team supporting research.

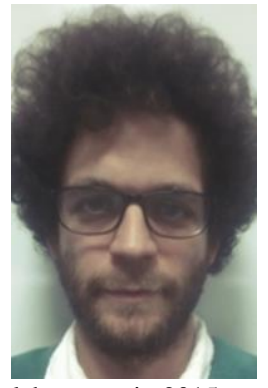

Simon Lambert received his $\mathrm{PhD}$ in physics from the University of Paris XI, France, in 2011. At that time, he was working on simulation and characterization of very small superconducting coils for mouse brain imaging. He was a postdoctoral fellow with the University of Paris Diderot, working at Beaujon Hospital in a clinical environment, from 2011 to 2013, on advanced methodological MRI developments for liver disease. He then continued as a postdoctoral fellow at King's College London on MR elastography and new diffusion sequences. He joined the University of Lyon 1 and the CREATIS laboratory in 2015 as assistant professor. His current research interests include radiofrequency coils, MR elastography, and new MR methods to characterized tissue microarchitecture.

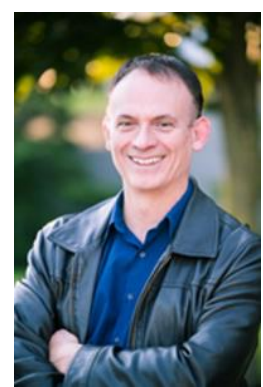

Fraser Robb graduated with a degree in Applied Physics from the University of Strathclyde (1990) and from the University of Aberdeen with an MSc and PhD in Medical Physics / MRI (1992/1998). For the past 19 years he has been working in the MRI industry, and for the last 17 years with GE Healthcare, Inc., where he is Chief Technology Leader for MRI Coils working on product development. He works closely with research customers around the world, developing best-in -class MRI technology.

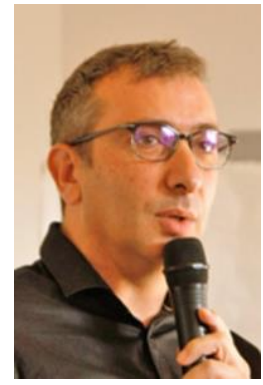

Olivier Beuf was born in France in 1970. He received his $\mathrm{PhD}$ degree in physics from the University of Lyon, France, in 1998. From 1998 to 2000, he was a postdoctoral fellow at the University of California, San Francisco, CA, USA working on advanced methodological MRI developments for musculoskeletal applications. His professional career includes a position as an MRI advanced application expert at Siemens Medical Solutions before joining the CNRS in 2000. Olivier Beuf was head of the NMR and optics team for more than 10 years and is now the director of the CREATIS laboratory. He has coauthored 90 publications, three patents, more than 200 communications, and has been involved in multiple international collaborations with academic and industrial partners. $\mathrm{He}$ has vast experience in clinical and preclinical applications of MRI as well as a thorough understanding of all the instrumental aspects that are required for the implementation of the considered solutions on MR systems. MR imaging, radiofrequency coils, multiparameters quantitative imaging (elastography, diffusion, etc.), liver examination, and cartilage ultra-structure and morphology are a few examples of his research interests. 